(C) 2017 IEEE. Personal use of this material is permitted. Permission from IEEE must be obtained for all other uses, in any current or future media, including reprinting/republishing this material for advertising or promotional purposes, creating new collective works, for resale or redistribution to servers or lists, or reuse of any copyrighted component of this work in other works. 


\title{
A Passivity Preserving Frequency-Weighted Model Order Reduction Technique
}

\author{
Umair Zulfiqar, Waseem Tariq, Li Li and Muwahida Liaquat
}

\begin{abstract}
Frequency-weighted model order reduction techniques aim to yield a reduced order model whose output matches that of the original system in the emphasized frequency region. However, passivity of the original system is only known to be preserved in the single-sided weighted case. A frequency-weighted model order reduction technique is proposed which guarantees the passive reduced models in the double-sided weighted case. A set of easily computable error bound expressions are also presented.
\end{abstract}

Index Terms-Error bound, Frequency-weighted Gramians, Model order reduction, Passivity.

\section{INTRODUCTION}

$\mathbf{M}$ ODEL order reduction (MOR) has been the attention of researchers for the past few decades. The aim of MOR is to find a fairly accurate lower order approximate model which retains the essential properties of the original system like stability, passivity and input-output behaviour [1]-[5]. Truncated balanced realization (TBR) [6] is one of the most popular MOR techniques due to its accuracy, preservation of stability and easily computable a priori error bound.

In the context of MOR of $R L C$ networks, integrated circuits, control systems and interconnected circuits, passivity is an important property to be preserved because non-passive reduced order model (ROM) may yield nonphysical behaviour by generating energy at high frequencies and resulting in erratic time-domain behaviours. Passivity implies stability but the opposite is not true. Philips et al. [7] extended TBR [6] to preserve passivity of the original system and also presented the corresponding error bound expressions.

In many practical applications, it is desirable that the output of ROM matches that of the original system in some specified frequency region [8]. Enns [9] presented a frequency-weighted generalization of TBR [6] for that purpose. However, stability is only guaranteed in the case of single-sided frequency weighting with no a priori error bound provided. Several modifications exist in the literature like [10]-[13] to ensure stability in the double-sided weighted case and error bounds are also derived. These techniques [10]-[13], however, do not guarantee passivity of the ROM.

Heydari and Pedram [14] proposed a frequency-weighted generalization of Philips et al.'s technique [7]. Similarly, Enns'

U. Zulfiqar, W. Tariq and M. Liaquat are with the Department of Electrical Engineering, College of Electrical and Mechanical Engineering, National University of Sciences and Technology (NUST), Islamabad 44000, Pakistan (email: umairzulfiqar79@ee.ceme.edu.pk; waseemtariq79@ee.ceme.edu.pk; muwahida@ee.ceme.edu.pk)

L. Li is with the School of Electrical, Mechanical and Mechatronic Systems, University of Technology Sydney, Sydney, Australia (email: li.li@uts.edu.au) technique [9] and its modifications [10]-[11] are generalized in [15] which claimed to yield the guaranteed passive ROMs. However, it is pointed in [16] that these techniques [14][15] guarantee the passivity only in the single-sided weighted case. Many approximation criteria in MOR are double-sided weighted criteria, particularly, when the model to be reduced is part of a closed-loop system and preservation of the closedloop behaviour is important [17]. Frequency-weighted MOR (FWMOR) techniques are used to satisfy these criteria and preservation of passivity in the double-sided weighted case is, therefore, critical.

To the authors' best knowledge, there is no FWMOR technique in the literature so far which guarantees the passivity in the double-sided weighted case. In this paper, an FWMOR technique is proposed which preserves passivity of the original system both in the single and double-sided weighted cases. A set of easily computable error bound expressions are also derived. Numerical examples are presented to show the efficacy of proposed technique.

\section{BACKGROUND}

Consider an $n^{\text {th }}$ order positive-real linear time invariant system

$$
G(s)=C(s I-A)^{-1} B+D
$$

where $\{A, B, C, D\}$ is its minimal state-space realization, $A \in$ $\mathbb{R}^{n \times n}, B \in \mathbb{R}^{n \times m}, C \in \mathbb{R}^{m \times n}$ and $D \in \mathbb{R}^{m \times m}$.

\section{A. Philips et al.'s Technique [7]}

The controllability Gramian-like matrix $P_{a}$ and the observability Gramian-like matrix $Q_{a}$ of the system $\{A, B, C, D\}$ are the solution of following Lur'e equations:

$$
\begin{array}{rlrl}
A P_{a}+P_{a} A^{T} & =-K_{c} K_{c}^{T} & & \\
P_{a} C^{T}-B & =-K_{c} J_{c}^{T} & & J_{c} J_{c}^{T}=D+D^{T} \\
A^{T} Q_{a}+Q_{a} A & =-K_{o}^{T} K_{o} & & \\
Q_{a} B-C^{T} & =-K_{o}^{T} J_{o} & J_{o}^{T} J_{o}=D+D^{T}
\end{array}
$$

The transformation matrix $T_{a}$ is calculated such that $T_{a}^{T} Q_{a} T_{a}=T_{a}^{-1} P_{a} T_{a}^{-T}=\operatorname{diag}\left\{\xi_{1}, \xi_{2}, \cdots, \xi_{n}\right\}$ and $\xi_{1} \geq$ $\xi_{2} \geq \cdots \geq \xi_{n}$. ROM is obtained by applying the transformation on the original system and truncating the transformed system up to the desired order.

Remark 1: The Lur'e equations in (1)-(4) can be solved using Riccati equation based algorithms in [18]. 


\section{B. Heydari and Pedram's Technique (HPT) [14]}

Let $V(s)$ and $W(s)$ be the positive-real transfer functions of the input and output frequency weights respectively.

$$
\begin{aligned}
V(s) & =\hat{C}_{v}\left(s I-\hat{A}_{v}\right)^{-1} \hat{B}_{v}+\hat{D}_{v} \\
W(s) & =\hat{C}_{w}\left(s I-\hat{A}_{w}\right)^{-1} \hat{B}_{w}+\hat{D}_{w}
\end{aligned}
$$

Then, the augmented systems are represented as the following:

$$
\begin{gathered}
G(s) V(s)=\left[\begin{array}{c|c}
A_{i} & B_{i} \\
\hline C_{i} & D_{i}
\end{array}\right]=\left[\begin{array}{cc|c}
A & B \hat{C}_{v} & B \hat{D}_{v} \\
0 & \hat{A}_{v} & \hat{B}_{v} \\
\hline C & D \hat{C}_{v} & D \hat{D}_{v}
\end{array}\right] \\
W(s) G(s)=\left[\begin{array}{c|c}
A_{o} & B_{o} \\
\hline C_{o} & D_{o}
\end{array}\right]=\left[\begin{array}{cc|c}
A & 0 & B \\
\hat{B}_{w} C & \hat{A}_{w} & \hat{B}_{w} D \\
\hline \hat{D}_{w} C & \hat{C}_{w} & \hat{D}_{w} D
\end{array}\right]
\end{gathered}
$$

Heydari and Pedram [14] considered $D=0$. The controllability Gramian-like matrix $P_{i}$ and observability Gramianlike matrix $Q_{o}$ of the augmented systems are the solution of following Lur'e equations:

$$
\begin{aligned}
A_{i} P_{i}+P_{i} A_{i}^{T} & =-K_{c, i} K_{c, i}^{T} & P_{i} C_{i}^{T} & =B_{i} \\
A_{o}^{T} Q_{o}+Q_{o} A_{o} & =-K_{o, o}^{T} K_{o, o} & & Q_{o} B_{o}=C_{o}^{T}
\end{aligned}
$$

The matrices $P_{i}, Q_{o}, K_{c, i}$ and $K_{o, o}$ can be partitioned as

$$
\begin{aligned}
P_{i} & =\left[\begin{array}{ll}
\bar{P}_{11} & \bar{P}_{12} \\
\bar{P}_{12}^{T} & \bar{P}_{22}
\end{array}\right] \quad Q_{o}=\left[\begin{array}{ll}
\bar{Q}_{11} & \bar{Q}_{12} \\
\bar{Q}_{12}^{T} & \bar{Q}_{22}
\end{array}\right] \\
K_{c, i} & =\left[\begin{array}{l}
\bar{K}_{c, 1} \\
\bar{K}_{c, 2}
\end{array}\right] \quad K_{o, o}=\left[\begin{array}{l}
\bar{K}_{o, 1} \\
\bar{K}_{o, 2}
\end{array}\right]
\end{aligned}
$$

The frequency-weighted controllability Gramian-like matrix $\bar{P}_{11}$ and frequency-weighted observability Gramian-like matrix $\bar{Q}_{11}$, corresponding to the original system, can be expressed as

$$
\begin{array}{ll}
A \bar{P}_{11}+\bar{P}_{11} A^{T}=-X & \bar{P}_{11} C^{T}=B \hat{D}_{v} \\
A^{T} \bar{Q}_{11}+\bar{Q}_{11} A=-Y & \bar{Q}_{11} B=C^{T} \hat{D}_{w}^{T}
\end{array}
$$

where

$$
\begin{aligned}
& X=B \hat{C}_{v} \bar{P}_{12}^{T}+\bar{P}_{12} \hat{C}_{v}^{T} B^{T}+\bar{K}_{c, 1} \bar{K}_{c, 1}^{T} \\
& Y=C^{T} \hat{B}_{w}^{T} \bar{Q}_{12}^{T}+\bar{Q}_{12} \hat{B}_{w} C+\bar{K}_{o, 1}^{T} \bar{K}_{o, 1}
\end{aligned}
$$

Inspired by Wang et al.'s technique [11], Heydari and Pedram [14] replaced the generally indefinite symmetric matrices $X$ and $Y$ with their positive semidefinite approximations $\hat{K}_{c} \hat{K}_{c}^{T}$ and $\hat{K}_{o}^{T} \hat{K}_{o}$ respectively to ensure passivity (similar to the ensured stability in Wang et al. [11]) where $\hat{K}_{c}=U|S|^{1 / 2}$ and $\hat{K}_{o}=|R|^{1 / 2} V^{T}$. R, S,U and $V$ are obtained by the eigenvalue decomposition of $X$ and $Y$ i.e. $X=U S U^{T}$ and $Y=V R V^{T}$ where $S=\operatorname{diag}\left\{s_{1}, s_{2}, \cdots, s_{n}\right\}$ and $R=\operatorname{diag}\left\{r_{1}, r_{2}, \cdots, r_{n}\right\}$.

$$
\begin{aligned}
A P_{h}+P_{h} A^{T} & =-\hat{K}_{c} \hat{K}_{c}^{T} & & P_{h} C^{T}=\hat{B} \\
A^{T} Q_{h}+Q_{h} A & =-\hat{K}_{o}^{T} \hat{K}_{o} & & \bar{Q}_{h} B=\hat{C}^{T}
\end{aligned}
$$

The transformation matrix $T_{h}$ is calculated such that $T_{h}^{T} Q_{h} T_{h}=T_{h}^{-1} P_{h} T_{h}^{-T}=\Sigma=\operatorname{diag}\left\{\bar{\xi}_{1}, \bar{\xi}_{2}, \cdots, \bar{\xi}_{n}\right\}$ and $\bar{\xi}_{1} \geq \bar{\xi}_{2} \geq \cdots \geq \bar{\xi}_{n} \geq 0$. The $r^{t h}$ order ROM $G_{r}(s)$ is obtained by applying the transformation on the original system and truncating the transformed system up to the desired order. The following error bound holds for HPT [14]:

$$
\begin{aligned}
& \left\|W(s)\left(G(s)-G_{r}(s)\right) V(s)\right\|_{\infty} \leq \\
& 2\|W(s) L\|_{\infty}\|K V(s)\|_{\infty} \sum_{k=r+1}^{n} \bar{\xi}_{k}
\end{aligned}
$$

where

$$
\begin{aligned}
L & =C V \operatorname{diag}\left\{\left|r_{1}\right|^{-\frac{1}{2}},\left|r_{2}\right|^{-\frac{1}{2}}, \cdots,\left|r_{j}\right|^{-\frac{1}{2}}, 0, \cdots, 0\right\} \\
K & =\operatorname{diag}\left\{\left|s_{1}\right|^{-\frac{1}{2}},\left|s_{2}\right|^{-\frac{1}{2}}, \cdots,\left|s_{i}\right|^{-\frac{1}{2}}, 0, \cdots, 0\right\} U^{T} B \\
i & =\operatorname{rank}[X] \text { and } j=\operatorname{rank}[Y] .
\end{aligned}
$$

\section{Discussion}

It is shown by Muda et al. in [16] that HPT [14] does not guarantee the passivity in the double-sided weighted case. The reason is that $P_{h}$ and $Q_{h}$ are obtained from two different systems i.e. $\{A, \hat{B}, C\}$ and $\{A, B, \hat{C}\}$ respectively and a transformation matrix $T_{h}$ is obtained which balances $P_{h}$ and $Q_{h}$. Then $T_{h}$ is applied on the original system $\{A, B, C\}$ and therefore $T_{h}$ does not balance $P_{a}$ and $Q_{a}$. Consequently, the results of Philips et al. [7] for passivity assurance can not be used and the resulting ROM may be non-passive as shown in [16]. Although not explicitly mentioned by Muda et al. [16], the techniques in [15] do not guarantee the passivity either for the same reason. "Extended Wang's technique" in [15] is actually similar to HPT [14]. It is also shown in [16] that the passivity is guaranteed in the single-sided weighted case for HPT [14] because in this case either $P_{a}$ or $Q_{a}$ is diagonalized and thus, the results of Philips et al. [7] for passivity assurance can be used. The techniques in [15] also guarantee the passivity in the single-sided weighted case for the same reason.

It is also claimed in [16] that HPT [14] does not even guarantee the stability and therefore, the error bound expression in (13) is not valid. However, we will now show that this criticism is incorrect.

Lemma 1: HPT [14] yields stable ROMs and the error bound expression in (13) is also valid.

Proof: Consider the triple $\left\{A_{t}, B_{t}, C_{t}\right\}=$ $\left\{T_{h}^{-1} A T_{h}, T_{h}^{-1} \hat{K}_{c}, \hat{K}_{o} T_{h}\right\}$. It can easily be noted that this triple satisfy the following Lyapunov equations:

$$
A_{t} \Sigma+\Sigma A_{t}^{T}=-B_{t} B_{t}^{T} \quad A_{t}^{T} \Sigma+\Sigma A_{t}=-C_{t}^{T} C_{t}
$$

where $\Sigma=T_{h}^{T} Q_{h} T_{h}=T_{h}^{-1} P_{h} T_{h}^{-T}=\operatorname{diag}\left\{\bar{\xi}_{1}, \bar{\xi}_{2}, \cdots, \bar{\xi}_{n}\right\}$ and $\bar{\xi}_{1} \geq \bar{\xi}_{2} \geq \cdots \geq \bar{\xi}_{n} \geq 0$. It can then be concluded from Appendix $\mathrm{C}$ of [19] that $A_{r}=\mathcal{P}_{r} A_{t} \mathcal{P}_{r}^{T}$ is Hurwitz, where $\mathcal{P}_{r}=\left[\begin{array}{ll}I_{r} & 0\end{array}\right]$ is the truncating projection matrix. Note that this $A_{r}$ is also the $A$-matrix of the ROMs. Thus, ROMs are guaranteed to be stable.

This result is, in fact, not new and can be found at several places in the literature like [19]-[21]. Majority of the stability preserving FWMOR techniques [10]-[13] rely on this result for stability assurance. We agree with Muda et al. [16] that the Lur'e equations of ROM yielded by HPT [14] 
do not reduce to Lyapunov equations but this is not the only way to prove the stability of ROM.

An important shortcoming of the techniques in [14]-[15] (which is not pointed out in [16]) is needed to be discussed first before proceeding to the actual solution. The FWMOR techniques in [14]-[15] use the augmented systems comprising of a series connection of the original and weighting systems. However, the augmented system may not be passive even if both the original and weighting systems are passive because the series interconnection of passive systems, in contrast with the parallel and feedback interconnection, is not always passive (see for instance [22]). Thus, $W(s) G(s)$ and $G(s) V(s)$ satisfy (5)-(6) (with $P_{i} \geq 0$ and $Q_{o} \geq 0$ ) only when the augmented realizations are passive which is not always the case. These techniques [14]-[15] are only guaranteed to work for a special case when the augmented systems are passive.

\section{MAIN WORK}

It is customary in the reduction of large scale $R L C$ networks that at the first stage Krylov methods are applied because these are computationally efficient. However, the ROMs yielded by Krylov methods are not compact. Therefore, in the next stage TBR [6] is used to obtain a compact ROM [7], [23]. Inspired by this staging concept, it is proposed to consider the double-sided weighted problem as a sequence of two single-sided weighted problems. In the first stage, the states which have the least share in the energy transfer within the frequency region emphasized by the input frequency weight are truncated. In the second stage, the states which have the least share in the energy transfer within the frequency region emphasized by the output frequency weight are truncated. The sequence of the stages can be swapped. The obtained ROM is passive due to the fact that passivity is guaranteed in the single-sided weighted case [16].

A passivity preserving mixed balancing algorithm is presented in [24] which requires one Lyapunov equation and one Riccati equation to be solved. Inspired by [24], it is proposed to calculate the weighted Gramians from the Lyapunov equations and unweighted Gramians-like matrices from the Lur'e equations. In this way, the condition on the frequency weights and augmented system to be passive is removed. This is particularly useful in the controller reduction problems when the plant is not passive. The weights and consequently the augmented system in this scenario may not be passive even if the controller is passive [17]. Therefore, the proposed technique is more general and have a wider range of applicability.

The controllability Gramian of the input augmented system $G(s) V(s)$ is the solution of following Lyapunov equation:

$$
A_{i} P_{c i}+P_{c i} A_{i}^{T}+B_{i} B_{i}^{T}=0
$$

$P_{c i}$ can be partitioned as $P_{c i}=\left[\begin{array}{ll}P_{11} & P_{12} \\ P_{12}^{T} & P_{22}\end{array}\right]$. Block $(1,1)$ of (14) can be written as

$$
A P_{11}+P_{11} A^{T}+X_{1}=0
$$

where

$$
X_{1}=B \hat{C}_{v} P_{12}^{T}+P_{12} \hat{C}_{v}^{T} B^{T}+B \hat{D}_{v} \hat{D}_{v}^{T} B^{T}
$$

The frequency-weighted controllability Gramian $P_{u}$ is the solution of following Lyapunov equation

$$
A P_{u}+P_{u} A^{T}+B_{u} B_{u}^{T}=0
$$

where the fictitious input matrix $B_{u}=\bar{U}_{1} \bar{S}_{1}^{\frac{1}{2}}$. The matrices $\bar{U}_{1}$ and $\bar{S}_{1}$ are obtained from the eigenvalue decomposition of symmetric indefinite matrix $X_{1}$ (as done in [12]) i.e. $X_{1}=\bar{U} \bar{S} \bar{U}^{T}=\left[\begin{array}{ll}\bar{U}_{1} & \bar{U}_{2}\end{array}\right]\left[\begin{array}{cc}\bar{S}_{1} & 0 \\ 0 & \bar{S}_{2}\end{array}\right]\left[\begin{array}{c}\bar{U}_{1}^{T} \\ \bar{U}_{2}^{T}\end{array}\right]$ where $\bar{S}_{1}=$ $\operatorname{diag}\left\{\bar{s}_{1}, \bar{s}_{2}, \cdots, \bar{s}_{l}\right\}, \bar{s}_{1} \geq \bar{s}_{2} \geq \cdots \geq \bar{s}_{l}>0$ and $l$ is the number of positive eigenvalues of $X_{1}$.

Let $Y_{1}=K_{o}^{T} K_{o}$ where $K_{o}$ is defined in (3). Then, the eigenvalue decomposition of $Y_{1}$ is $Y_{1}=\bar{V} \bar{R} \bar{V}^{T}$ where $\bar{R}$ $=\operatorname{diag}\left\{\bar{r}_{1}, \bar{r}_{2}, \cdots, \bar{r}_{n}\right\}$. The fictitious output matrix $C_{f}$ can be defined as $C_{f}=\bar{R}^{\frac{1}{2}} \bar{V}^{T}$. $Q_{a}$ (in (3)) can be rewritten as a solution of following Lyapunov equation:

$$
A^{T} Q_{a}+Q_{a} A+C_{f}^{T} C_{f}=0
$$

$P_{u}$ and $Q_{a}$ are the controllability Gramian and observability Gramian respectively of the system $\left\{A, B_{u}, C_{f}, D\right\}$ similar to FWBT problems [10]-[13] (and similar to [12] in particular). The transformation matrix $T_{1}$ is calculated such that $T_{1}^{T} Q_{a} T_{1}=T_{1}^{-1} P_{u} T_{1}^{-T}=\bar{\Sigma}=\operatorname{diag}\left\{\bar{\sigma}_{1}, \bar{\sigma}_{2}, \cdots, \bar{\sigma}_{n}\right\}$ and $\bar{\sigma}_{1} \geq \bar{\sigma}_{2} \geq \cdots \geq \bar{\sigma}_{n}$. The transformed system $\{\bar{A}, \bar{B}, \bar{C}, \bar{D}\}=\left\{T_{1}^{-1} A T_{1}, T_{1}^{-1} B, C T_{1}, D\right\}$ is then given by

$$
\left[\begin{array}{c|c}
T_{1}^{-1} A T_{1} & T_{1}^{-1} B \\
\hline C T_{1} & D
\end{array}\right]=\left[\begin{array}{cc|c}
A_{r_{1}} & A_{12} & B_{r_{1}} \\
A_{21} & A_{22} & B_{2} \\
\hline C_{r_{1}} & C_{2} & D
\end{array}\right] .
$$

Then $G_{r_{1}}(s)=C_{r_{1}}\left(s I-A_{r_{1}}\right)^{-1} B_{r_{1}}+D$ is the $r_{1}^{t h}\left(r_{1}<r\right)$ order ROM such that $A_{r_{1}} \in \mathbb{R}^{r_{1} \times r_{1}}, B_{r_{1}} \in \mathbb{R}^{r_{1} \times m}$ and $C_{r_{1}} \in \mathbb{R}^{m \times r_{1}}$.

Theorem 1: The following a priori error bound holds for this stage of reduction if $\operatorname{rank}\left[\begin{array}{ll}B_{u} & B\end{array}\right]=\operatorname{rank}\left[B_{u}\right]$ and $\operatorname{rank}\left[\begin{array}{l}C_{f} \\ K_{c}\end{array}\right]=\operatorname{rank}\left[C_{f}\right]$ :

$$
\left\|\left(G(s)-G_{r_{1}}(s)\right) V(s)\right\|_{\infty} \leq 2\left\|L_{1}\right\|_{\infty}\left\|K_{1} V(s)\right\|_{\infty} \sum_{k=r_{1}+1}^{n} \bar{\sigma}_{k}
$$

where

$$
\begin{aligned}
L_{1} & =C \bar{V} \operatorname{diag}\left\{\bar{r}_{1}^{-\frac{1}{2}}, \bar{r}_{2}^{-\frac{1}{2}}, \cdots, \bar{r}_{\bar{j}}^{-\frac{1}{2}}, 0, \cdots, 0\right\} \\
K_{1} & =\operatorname{diag}\left\{\bar{s}_{1}^{-\frac{1}{2}}, \bar{s}_{2}^{-\frac{1}{2}}, \cdots, \bar{s}_{\bar{i}}^{-\frac{1}{2}}, 0, \cdots, 0\right\} \bar{U}^{T} B \\
\bar{i} & =\operatorname{rank}\left[X_{1}\right] \text { and } \bar{j}=\operatorname{rank}\left[Y_{1}\right] .
\end{aligned}
$$

Proof: The proof is similar to the error bound expressions in [11]-[13] and hence omitted.

Proposition 1: $\operatorname{ROM}\left\{A_{r_{1}}, B_{r_{1}}, C_{r_{1}}, D\right\}$ is passive. Proof: $\{\bar{A}, \bar{B}, \bar{C}, \bar{D}\}$ is passive since it is just the similarity 
transformation of $\{A, B, C, D\}$ and therefore, it satisfies the following Lur'e equations such that $\bar{\Sigma} \geq 0$ [7].

$$
\begin{aligned}
\bar{A}^{T} \bar{\Sigma}+\bar{\Sigma} \bar{A} & =-\bar{K}_{o}^{T} \bar{K}_{o} \\
\bar{\Sigma} \bar{B}-\bar{C}^{T} & =-\bar{K}_{o}^{T} J_{o} \quad J_{o}^{T} J_{o}=D+D^{T}
\end{aligned}
$$

$\bar{\Sigma}$ can be partitioned as $\operatorname{diag}\left\{\bar{\Sigma}_{r_{1}}, \bar{\Sigma}_{\left(n-r_{1}\right)}\right\}$ where $\bar{\Sigma}_{r_{1}}=$ $\operatorname{diag}\left\{\bar{\sigma}_{1} \geq \bar{\sigma}_{2} \geq \cdots \geq \bar{\sigma}_{r_{1}}\right\}$. $\operatorname{Block}(1,1)$ of $(20)-(21)$ can be written as

$$
\begin{aligned}
A_{r_{1}}^{T} \bar{\Sigma}_{r_{1}}+\bar{\Sigma}_{r_{1}} A_{r_{1}} & =-\bar{K}_{r_{1}}^{T} \bar{K}_{r_{1}} \\
\bar{\Sigma}_{r_{1}} B_{r_{1}}-C_{r_{1}}^{T} & =-\bar{K}_{r_{1}}^{T} J_{o} \quad J_{o}^{T} J_{o}=D+D^{T}
\end{aligned}
$$

$\left\{A_{r_{1}}, B_{r_{1}}, C_{r_{1}}, D\right\}$ satisfies positive-real lemma since $\bar{\Sigma}_{r_{1}} \geq 0$ (as $\left.\bar{\Sigma} \geq 0\right)$. Hence, $G_{r_{1}}(s)$ is passive.

Consider the output augmented system $W(s) G_{r_{1}}(s)$ which is represented in the state-space form as:

$$
W(s) G_{r_{1}}(s)=\left[\begin{array}{c|c}
\tilde{A}_{o} & \tilde{B}_{o} \\
\hline \tilde{C}_{o} & \tilde{D}_{o}
\end{array}\right]=\left[\begin{array}{cc|c}
A_{r_{1}} & 0 & B_{r_{1}} \\
\hat{B}_{w} C_{r_{1}} & \hat{A}_{w} & \hat{B}_{w} D \\
\hline \hat{D}_{w} C_{r_{1}} & \hat{C}_{w} & \hat{D}_{w} D
\end{array}\right]
$$

The observability Gramian of the output augmented system $W(s) G_{r_{1}}(s)$ is the solution of following Lyapunov equation:

$$
\tilde{A}_{o}^{T} Q_{o o}+Q_{o o} \tilde{A}_{o}+\tilde{C}_{o}^{T} \tilde{C}_{o}=0
$$

$Q_{o o}$ can be partitioned as $Q_{o o}=\left[\begin{array}{ll}Q_{11} & Q_{12} \\ Q_{12}^{T} & Q_{22}\end{array}\right]$. Block $(1,1)$ of (22) can be written as

$$
A_{r_{1}}^{T} Q_{11}+Q_{11} A_{r_{1}}+Y_{2}=0
$$

where

$$
Y_{2}=C_{r_{1}}^{T} \hat{B}_{w}^{T} Q_{12}^{T}+Q_{12} \hat{B}_{w} C_{r_{1}}+C_{r_{1}}^{T} \hat{D}_{w}^{T} \hat{D}_{w} C_{r_{1}}
$$

The frequency-weighted observability Gramian $Q_{u}$ is the solution of following Lyapunov equation

$$
A_{r_{1}}^{T} Q_{u}+Q_{u} A_{r_{1}}+C_{u}^{T} C_{u}=0
$$

where the fictitious output matrix $C_{u}=\tilde{R}_{1}^{\frac{1}{2}} \tilde{V}_{1}^{T}$. The matrices $\tilde{V}_{1}$ and $\tilde{R}_{1}$ are obtained from the eigenvalue decomposition of symmetric indefinite matrix $Y_{2}$ (as done in [12]) i.e. $Y_{2}=\tilde{V} \tilde{R} \tilde{V}^{T}=\left[\begin{array}{ll}\tilde{V}_{1} & \tilde{V}_{2}\end{array}\right]\left[\begin{array}{cc}\tilde{R}_{1} & 0 \\ 0 & \tilde{R}_{2}\end{array}\right]\left[\begin{array}{c}\tilde{V}_{1}^{T} \\ \tilde{V}_{2}^{T}\end{array}\right]$ where $\tilde{R}_{1}=$ $\operatorname{diag}\left\{\tilde{r}_{1}, \tilde{r}_{2}, \cdots, \tilde{r}_{\tilde{l}}\right\}, \tilde{r}_{1} \geq \cdots \geq \tilde{r}_{\tilde{l}}>0$ and $\tilde{l}$ is the number of positive eigenvalues of $Y_{2}$.

The controllability Gramian-like matrix $\tilde{P}_{a}$ of the system $G_{r_{1}}(s)$ is the solution of following Lur'e equation:

$$
\begin{aligned}
A_{r_{1}} \tilde{P}_{a}+\tilde{P}_{a} A_{r_{1}}^{T} & =-\tilde{K}_{c} \tilde{K}_{c}^{T} \\
\tilde{P}_{a} C_{r_{1}}^{T}-B_{r_{1}} & =-\tilde{K}_{c} \tilde{J}_{c}^{T} \quad \tilde{J}_{c} \tilde{J}_{c}^{T}=D+D^{T}
\end{aligned}
$$

Similar to the previous stage, let $X_{2}=\tilde{K}_{c} \tilde{K}_{c}^{T}$. Then, the eigenvalue decomposition of $X_{2}$ is $X_{2}=\tilde{U} \tilde{S} \tilde{U}^{T}$ where $\tilde{S}=$ $\operatorname{diag}\left\{\tilde{s}_{1}, \tilde{s}_{2}, \cdots, \tilde{s}_{n}\right\}$. The fictitious input matrix $B_{f}$ can be defined as $B_{f}=\tilde{U} \tilde{S}^{\frac{1}{2}}$. $\tilde{P}_{a}$ (in (26)) can be rewritten as a solution of following Lyapunov equation:

$$
A_{r_{1}} \tilde{P}_{a}+\tilde{P}_{a} A_{r_{1}}+B_{f} B_{f}^{T}=0
$$

The transformation matrix $T_{2}$ is calculated such that $T_{2}^{T} Q_{u} T_{2}=T_{2}^{-1} \tilde{P}_{a} T_{2}^{-T}=\tilde{\Sigma}^{\tilde{\Sigma}}=\operatorname{diag}\left\{\tilde{\sigma}_{1}, \tilde{\sigma}_{2}, \cdots, \tilde{\sigma}_{r_{1}}\right\}$ and $\tilde{\sigma}_{1} \geq \tilde{\sigma}_{2} \geq \cdots \geq \tilde{\sigma}_{r_{1}}$. The transformed system $\{\tilde{A}, \tilde{B}, \tilde{C}, \tilde{D}\}=\left\{T_{2}^{-1} A_{r_{1}} T_{2}, T_{2}^{-1} B_{r_{1}}, C_{r_{1}} T_{2}, D\right\}$ is then given by

$$
\left[\begin{array}{c|c}
T_{2}^{-1} A_{r_{1}} T_{2} & T_{2}^{-1} B_{r_{1}} \\
\hline C_{r_{1}} T_{2} & D
\end{array}\right]=\left[\begin{array}{cc|c}
A_{r} & A_{r_{12}} & B_{r} \\
A_{r_{21}} & A_{r_{22}} & B_{r_{2}} \\
\hline C_{r} & C_{r_{2}} & D
\end{array}\right]
$$

where $G_{r}(s)=C_{r}\left(s I-A_{r}\right)^{-1} B_{r}+D$ is the $r^{\text {th }}$ order ROM such that $A_{r} \in \mathbb{R}^{r \times r}, B_{r} \in \mathbb{R}^{r \times m}$ and $C_{r} \in \mathbb{R}^{m \times r}$. The following error bound holds for this stage of reduction if $\operatorname{rank}\left[\begin{array}{ll}B_{f} & \tilde{K}_{c}\end{array}\right]=\operatorname{rank}\left[B_{f}\right]$ and $\operatorname{rank}\left[\begin{array}{c}C_{u} \\ C_{r_{1}}\end{array}\right]=\operatorname{rank}\left[C_{u}\right]$ :

$\left\|W(s)\left(G_{r_{1}}(s)-G_{r}(s)\right)\right\|_{\infty} \leq 2\left\|W(s) L_{2}\right\|_{\infty}\left\|K_{2}\right\|_{\infty} \sum_{k=r+1}^{r_{1}} \tilde{\sigma}_{k}$ where

$$
\begin{aligned}
L_{2} & =C_{r_{1}} \tilde{V} \operatorname{diag}\left\{\tilde{r}_{1}^{-\frac{1}{2}}, \tilde{r}_{2}^{-\frac{1}{2}}, \cdots, \tilde{r}_{\tilde{j}}^{-\frac{1}{2}}, 0, \cdots, 0\right\} \\
K_{2} & =\operatorname{diag}\left\{\tilde{s}_{1}^{-\frac{1}{2}}, \tilde{s}_{2}^{-\frac{1}{2}}, \cdots, \tilde{s}_{\tilde{i}}^{-\frac{1}{2}}, 0, \cdots, 0\right\} \tilde{U}^{T} B_{r_{1}} \\
\tilde{i} & =\operatorname{rank}\left[X_{2}\right] \text { and } \tilde{j}=\operatorname{rank}\left[Y_{2}\right] .
\end{aligned}
$$

The overall error bound can be expressed as:

$$
\begin{gathered}
\left\|W(s)\left(G(s)-G_{r}(s)\right) V(s)\right\|_{\infty}= \\
\left\|W(s)\left(G(s)-G_{r_{1}}(s)+G_{r_{1}}(s)-G_{r}(s)\right) V(s)\right\|_{\infty} \leq \\
\|W(s)\|_{\infty}\left\|\left(G(s)-G_{r_{1}}(s)\right) V(s)\right\|_{\infty}+ \\
\left\|W(s)\left(G_{r_{1}}(s)-G_{r}(s)\right)\right\|_{\infty}\|V(s)\|_{\infty} \leq \\
2\|W(s)\|_{\infty}\left\|L_{1}\right\|_{\infty}\left\|K_{1} V(s)\right\|_{\infty} \sum_{k=r_{1}+1}^{n} \bar{\sigma}_{k}+ \\
2\|V(s)\|_{\infty}\left\|W(s) L_{2}\right\|_{\infty}\left\|K_{2}\right\|_{\infty} \sum_{k_{1}=r+1}^{r_{1}} \tilde{\sigma}_{k_{1}}
\end{gathered}
$$

Theorem 2: $\operatorname{ROM}\left\{A_{r}, B_{r}, C_{r}, D\right\}$ is passive.

Proof: Proof is similar to that of Proposition 1 and hence omitted.

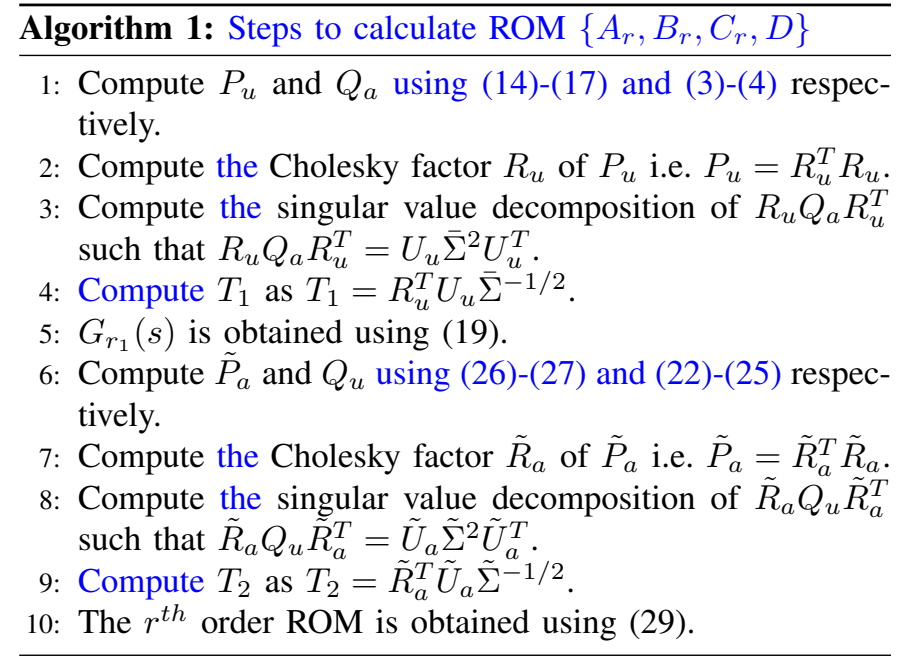

Remark 2: The sequence of the stages can be swaped, i.e. the first stage can be output-weighted stage and the second 
stage can be input-weighted stage or vice versa.

Remark 3: ROMs yielded by the proposed technique are not unique. Therefore, the parameter $r_{1}\left(r<r_{1}<n\right)$ can be adjusted to achieve the best results. In the single-sided weighted case, $r_{1}=r$ (for input weighting only) or $r_{1}=n$ (for output weighting only).

\section{NuMERICAL EXAMPLES}

In this section, two passive models of a wire comprising of 100 and 150 repeated $R L C$ sections are considered which produce a $200^{t h}$ and $300^{\text {th }}$ order system respectively [23]. The input and output weighting systems considered are

$$
V(s)=\frac{10}{(s+10)} \quad W(s)=\frac{(s+0.01)}{(s+0.05)}
$$

The models are reduced using HPT [14], Extended Enns' technique (EET) [15], Extended Lin and Chiu's technique (ELCT) [15] and the proposed technique. Although HPT [14], EET [15] and ELCT [15] do not guarantee the passive ROMs, these do not always yield non-passive models. $r_{1}$ is not changed during the experiment for a fair comparison i.e. $r_{1}=100$ in the first case and $r_{2}=150$ in the second case. Table I compares the weighted error $\left\|W(s)\left(G(s)-G_{r}(s)\right) V(s)\right\|_{\infty}$ (magnified by the factor of $10^{5}$ ) of the ROMs yielded by these techniques. As shown, the proposed technique guarantees passivity of the ROMs without any considerable increase in error.

TABLE I: Error Comparison

\begin{tabular}{|c|c|c|c|c|c|}
\hline \multirow{3}{*}{ Example } & & \multicolumn{4}{|c|}{$\left\|W(s)\left(G(s)-G_{r}(s)\right) V(s)\right\|_{\infty} \times 10^{5}$} \\
\cline { 2 - 6 } & Order & HPT [14] & EET [15] & ELCT [15] & Proposed \\
\hline \multirow{4}{*}{1} & 11 & 1.1988 & 0.7133 & 0.7710 & 0.3773 \\
\cline { 2 - 6 } & 12 & 0.0651 & 0.0653 & 0.0702 & 0.0328 \\
\cline { 2 - 6 } & 13 & 0.0571 & 0.0524 & 0.0581 & 0.0325 \\
\cline { 2 - 6 } & 14 & 0.0250 & 0.0152 & 0.0164 & 0.0070 \\
\cline { 2 - 6 } & 15 & 0.0012 & 0.0171 & 0.0033 & 0.0080 \\
\hline \multirow{4}{*}{2} & 16 & 0.0016 & 0.0138 & 0.0052 & 0.0077 \\
\cline { 2 - 6 } & 17 & 0.0018 & 0.0353 & 0.0044 & 0.0075 \\
\cline { 2 - 6 } & 18 & 0.0017 & 0.0136 & 0.0044 & 0.0075 \\
\cline { 2 - 6 } & 19 & 0.0017 & 0.0135 & 0.0044 & 0.0076 \\
\cline { 2 - 6 } & 20 & 0.0017 & 0.0135 & 0.0043 & 0.0076 \\
\hline
\end{tabular}

\section{CONCLuSion}

In this paper, some limitations in the existing FWMOR techniques are first highlighted. Then, an FWMOR technique is proposed which considers the double-sided weighted problem as a sequence of two single-sided problems and addresses these limitations. Easily computable error bound expressions are also presented. It is shown with the help of numerical examples that the proposed technique compares well in accuracy with the existing FWMOR techniques (which do not guarantee the passivity). However, the ROMs yielded by the proposed technique are guaranteed to be passive.

\section{REFERENCES}

[1] M. Imran and A. Ghafoor, "Stability preserving model reduction technique and error bounds using frequency-limited gramians for discretetime systems," IEEE Transactions on Circuits and Systems II: Express Briefs, vol. 9, no. 61, pp. 716-720, 2014.
[2] H. Liu, L. Daniel, and N. Wong, "Model reduction and simulation of nonlinear circuits via tensor decomposition," IEEE Transactions on Computer-Aided Design of Integrated Circuits and Systems, vol. 34, no. 7, pp. 1059-1069, 2015.

[3] E. R. Samuel, L. Knockaert, and T. Dhaene, "Model order reduction of time-delay systems using a laguerre expansion technique," IEEE Transactions on Circuits and Systems I: Regular Papers, vol. 61, no. 6, pp. 1815-1823, 2014.

[4] A. Davoudi, J. Jatskevich, P. L. Chapman, and A. Bidram, "Multiresolution modeling of power electronics circuits using model-order reduction techniques," IEEE Transactions on Circuits and Systems I: Regular Papers, vol. 60, no. 3, pp. 810-823, 2013.

[5] E. R. Samuel, L. Knockaert, and T. Dhaene, "Matrix-interpolation-based parametric model order reduction for multiconductor transmission lines with delays," IEEE Transactions on Circuits and Systems II: Express Briefs, vol. 62, no. 3, pp. 276-280, 2015.

[6] B. Moore, "Principal component analysis in linear systems: Controllability, observability, and model reduction," IEEE transactions on automatic control, vol. 26, no. 1, pp. 17-32, 1981.

[7] J. R. Phillips, L. Daniel, and L. M. Silveira, "Guaranteed passive balancing transformations for model order reduction," IEEE Transactions on Computer-Aided Design of Integrated Circuits and Systems, vol. 22, no. 8, pp. 1027-1041, 2003.

[8] U. Zulfiqar, M. Imran, A. Ghafoor, and M. Liaquat, "A new frequency limited interval gramian based model order reduction technique," IEEE Transactions on Circuits and Systems II: Express Briefs, 2016.

[9] D. F. Enns, "Model reduction with balanced realizations: An error bound and a frequency weighted generalization," in Decision and Control, 1984. The 23rd IEEE Conference on. IEEE, 1984, pp. 127-132.

[10] C.-A. Lin, T.-Y. Chiu et al., "Model-reduction via frequency weighted balanced realization," Control-Theory and Advanced Technology, vol. 8, no. 2, pp. 341-351, 1992.

[11] G. Wang, V. Sreeram, W. Liu et al., "A new frequency-weighted balanced truncation method and an error bound," IEEE Transactions on Automatic Control, vol. 44, no. 9, pp. 1734-1737, 1999.

[12] A. Varga and B. D. Anderson, "Accuracy-enhancing methods for balancing-related frequency-weighted model and controller reduction," Automatica, vol. 39, no. 5, pp. 919-927, 2003.

[13] M. Imran, A. Ghafoor, and V. Sreeram, "A frequency weighted model order reduction technique and error bounds," Automatica, vol. 50, no. 12, pp. 3304-3309, 2014.

[14] P. Heydari and M. Pedram, "Model-order reduction using variational balanced truncation with spectral shaping," IEEE Transactions on Circuits and Systems I: Regular Papers, vol. 53, no. 4, pp. 879-891, 2006.

[15] W. M. W. Muda, V. Sreeram, and H. H.-C. Lu, "Passivity-preserving frequency weighted model order reduction techniques for general largescale rlc sytems," in 2010 11th International Conference on Control Automation Robotics\&Vision, 2010.

[16] W. M. W. Muda, V. Sreeram, M. B. Ha, and A. Ghafoor, "Comments on model-order reduction using variational balanced truncation with spectral shaping," IEEE Transactions on Circuits and Systems I: Regular Papers, vol. 62, no. 1, pp. 333-335, 2015.

[17] G. Obinata and B. D. Anderson, Model reduction for control system design. Springer Science \& Business Media, 2012.

[18] N. Sadegh, J. D. Finney, and B. S. Heck, "An explicit method for computing the positive real lemma matrices," International Journal of Robust and Nonlinear Control, vol. 7, no. 12, pp. 1057-1069, 1997.

[19] W. Gawronski and J.-N. Juang, "Model reduction in limited time and frequency intervals," International Journal of Systems Science, vol. 21, no. 2, pp. 349-376, 1990.

[20] K. Zhou, J. C. Doyle, K. Glover et al., Robust and optimal control. Prentice hall New Jersey, 1996, vol. 40.

[21] S. Gugercin and A. C. Antoulas, "A survey of model reduction by balanced truncation and some new results," International Journal of Control, vol. 77, no. 8, pp. 748-766, 2004.

[22] Series interconnection of passive systems. [Online]. Available: http://www.mathworks.com/help/control/ug/series-interconnectionof-passive-systems.html

[23] N. Wong, V. Balakrishnan, C.-K. Koh, and T.-S. Ng, "Two algorithms for fast and accurate passivity-preserving model order reduction," IEEE Transactions on Computer-Aided Design of Integrated Circuits and Systems, vol. 25, no. 10, pp. 2062-2075, 2006.

[24] K. Unneland, P. Van Dooren, and O. Egeland, "New schemes for positive real truncation," Modeling, Identification and Control, vol. 28, no. 3, pp. 53-67, 2007. 